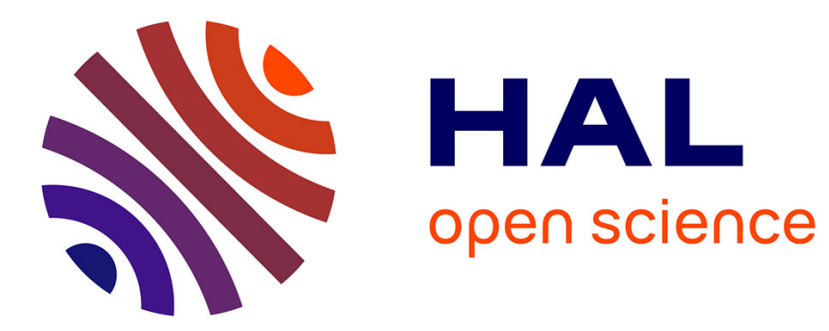

\title{
Simulation numérique du contrôle acoustique actif appliqué au bruit d'un turboréacteur
}

\author{
D. Berge, J. Cailleau
}

\section{To cite this version:}

D. Berge, J. Cailleau. Simulation numérique du contrôle acoustique actif appliqué au bruit d'un turboréacteur. Journal de Physique IV Proceedings, 1994, 04 (C5), pp.C5-199-C5-202. 10.1051/jp4:1994538 . jpa-00253033

\section{HAL Id: jpa-00253033 https://hal.science/jpa-00253033}

Submitted on 1 Jan 1994

HAL is a multi-disciplinary open access archive for the deposit and dissemination of scientific research documents, whether they are published or not. The documents may come from teaching and research institutions in France or abroad, or from public or private research centers.
L'archive ouverte pluridisciplinaire HAL, est destinée au dépôt et à la diffusion de documents scientifiques de niveau recherche, publiés ou non, émanant des établissements d'enseignement et de recherche français ou étrangers, des laboratoires publics ou privés. 


\title{
Simulation numérique du contrôle acoustique actif appliqué au bruit d'un turboréacteur
}

\author{
D. BERGE et J.M. CAILLEAU
}

SNECMA Villaroche, 77550 Moissy Cramayel, France

\begin{abstract}
Jet engine technology in the years to come will focus on Ultra-High Bypass Ratio concepts (UHBR), i.e. low speed fan design associated with a relatively low-number of fan blades. A direct consequence will be an emphasis on low frequencies in the flyover noise signature, exactly where current "passive" noise reduction solutions tend to be less effective and exhibit serious limitations, not mentionning the ever shorter nacelle dimension associated with those new engine concepts. In the world where increasing noise limits stringency is more and more often considered as a way to reduce aircraft noise, engine manufacturers had to take real steps towards new noise reduction technology and as a consequence get interested in the potential applications of active noise control. Our prime objective is to be able to control the directivity of engine at low frequency radiation. Accordingly one of the ways, the goal is not to reduce the acoustical field in all the directions, but only in priviliged ones with the concentration of acoustical energy in a non inconvenient domain. To validate this concept, a numerical simulation technique has been set up. After a presentation of the method employed, priliminary results are analysed with and without simulated active noise control.
\end{abstract}

\section{Introduction.}

Les moteurs d'avions, que conçoit et fabrique SNECMA (FRANCE) doivent respecter les normes antipollutions. C'est pourquoi la limitation du bruit est une priorite importante pour que nos produits satisfassent les réglementations en cours et surtout anticipent celles à venir. A l'heure actuelle, les moyens mis en oeuvre ( conception silencieuse, traitements acoustiques passifs) permettent de réduire suffisamment la signature acoustique du moteur.

Cependant, nous connaissons déjà certaines tendances des futurs moteurs d'avions civils: vitesse de rotation plus faible, moins de pales sur le fan. Les fréquences émises baisseront et nécessiteront des traitements acoustiques qui, pour rester efficaces, auront un encombrement (poids, épaisseur) imcompatible avec la place disponible. Les normes devenant de plus en plus sévères, tout porte à chercher des solutions pour remplacer ou compléter le traitement acoustique des moteurs.

Le contrôle acoustique actif fait partie des différents axes de recherche de SNECMA et semble comporter,du moins dans le principe, un certain nombre d'atouts pour aboutir à de bons résultats. Mais beaucoup d'obstacles restent à être franchis, en pensant notamment au problème de puissance des sources secondaires.

Le laboratoire de recherche en acoustique de SNECMA a engagé une double action en menant simultanément des études théoriques et expérimentales. Le présent papier porte sur les études théoriques et expose la méthode adoptée pour réaliser une simulation numérique du contrôle acoustique actif. 


\section{La simulation numérique.}

\subsection{Position du problème.}

Le problème qui se pose est complexe car le domaine où il est nécessaire de limiter le bruit (riverains au sol, passagers) n'est pas celui où il est possible de disposer les microphones de contrôle. Une des priorités sera donc d'observer et de maîtriser la répercussion sur le champ lointain du contrôle actif d'un champ acoustique d'une grande richesse modale situé à linterieur ou à proximité du moteur. Plusieurs voies sont possibles pour simuler numériquement le Contrôle Acoustique Actif (CAA). Cependant, selon les objectifs, il faut poser les bonnes hypothèses. Notre souhait étant de se rendre capable de calculer le champ acoustique avec puis sans CAA pour une configuration donnée de fonctionnement (position et nombre de points de contrôle et de sources secondaires) et ayant un souci de simplification, la première hypothèse s'imposant est la stationnarité du champ acoustique dans le temps. Il nous suffit dès lors de pouvoir calculer dans tout un domaine fluide ou sur des parois le champ acoustique complexe rayonné par différents types de sources. Enfin, un critère de minimisation permet de configurer le champ secondaire par des commandes appropriées de telle sorte que le champ total soit conforme aux attentes.

\subsection{Calcul du rayonnement acoustique.}

L' ERASCA ( Environnement sur la base du code Rayon-Axi pour la Simulation du Contrôle Actif) s'articule autour du code Rayon-Axi développé par STRACO (Compiègne-France). A partir d'une formulation variationelle, ce code résout les équations intégrales de l'acoustique par la méthode des éléments finis de frontière [1] [2]. Il permet par conséquent de calculer en tout point d'un domaine tridimensionnel la pression ou la vitesse acoustique. Il faut remarquer que cette version du code Rayon nécessite une définition axisymétrique de la géométrie. Cela impose alors un calcul du rayonnement acoustique mode par mode, fréquence par fréquence, et source par source (sans couplage), mais permet une forte réduction du temps de calcul. Enfin, il n'est pas pris en compte ni écoulement ni ni bruit large bande. Les points de calcul sont donc situés d'une part près du moteur, ils serviront de points de contrôle, et d'autre part au loin, dans le domaine nécessitant une réduction du bruit, ce seront les points d'observation (fig. 1).

\subsection{Simulation des sources.}

Dans un premier temps, et sans souci de validation, deux types de sources ont été mis au point:

\subsubsection{Source étendue.}

Elle sert de source primaire. La définition de cette source consiste à imposer un champ de pression sur une surface plane. Cette surface est généralement une section du conduit moteur. Cette définition, établie par mode azimutal, prend en compte les modes radiaux passants.

\subsubsection{Sources locales.}

Elles servent de sources secondaires. La définition d'une source locale consiste à imposer une pression complexe sur un élément de surface du conduit. Initialement, cette pression complexe est égale à $(1 ., 0$.).

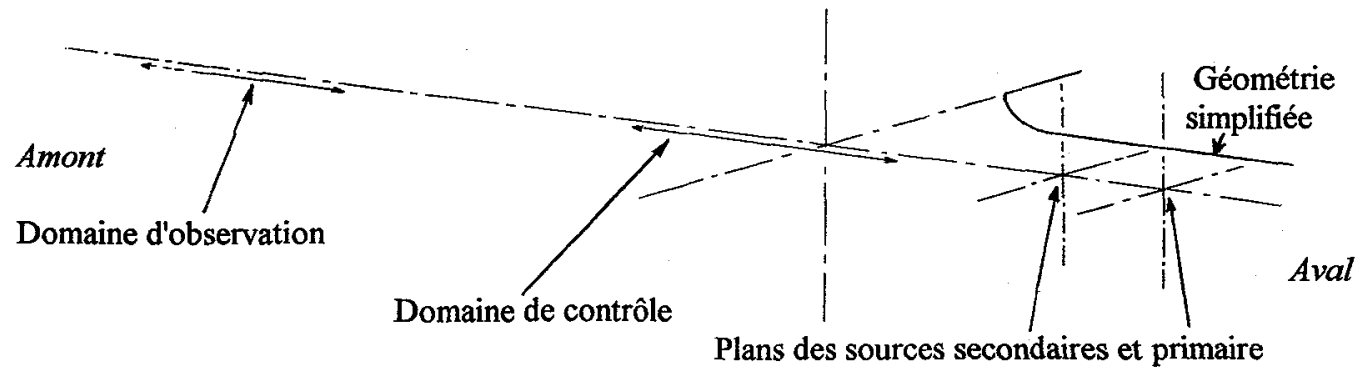

fig. 1 


\subsection{Le critère de minimisation.}

Au i ieme point de calcul $P_{i}$ du domaine de contrôle, il est donc possible de calculer la contribution des différentes sources, à savoir :

$$
\begin{gathered}
\text { a celle relative à la source primaire, notée } \quad P_{i}^{n}(p) \\
\text { a celle relative à la jieme source secondaire,notée } \\
\text { En } \mathbf{P i} \text {, la pression acoustique sera donc }
\end{gathered} \quad P_{i j}^{n}=U_{j}^{n} \cdot H_{i j}^{n} \quad P_{i}^{n}=P_{i}^{n}(p)+U_{j}^{n} \cdot H_{i j}^{n}
$$

Notre choix s'est porté sur une minimisation quadratique de l'énergie totale calculée sur l'ensemble des $\mathrm{i}$ points de contrôle, ce qui donne dans le domaine fréquentiel $\frac{\partial e}{\partial u_{j}^{n}}=0$

avec $e=\sum_{n=1, N} P_{i}^{n} \cdot P_{i}^{n^{*}}=\sum_{i}\left\{\underset{n}{\sum}\left(P_{i}^{n}(p)+\sum_{j} U_{j}^{n_{i j}^{n}} H_{i j}\right)\left(P_{i}(p)+\sum_{j j} U_{j j}^{n^{*} H_{i j}^{*}}\right)\right\}$

$$
\frac{\partial e}{\partial u_{j j}^{*}}=0
$$

Comme $\mathrm{j}$ ou jj sont des indices muets, l'équation (4) est la transposée de l'équation (5). Elles ont donc les mêmes solutions. En intégrant l'équation (6) dans (5), il vient :

$$
\sum_{i}^{\sum\left(H_{i j j}^{n^{*}}\left(P_{i}^{n}+\sum_{j} U_{j}^{n} \cdot H_{i j}\right)\right.}=0 \quad \text { avec } 1 \leq \mathrm{i} \leq \mathrm{I} \text { et } 1 \leq \mathrm{jj} \leq \mathrm{J}
$$

Il y a deux façons de déterminer les solutions de (7) :

a en résolvant le système $A_{i}=0$

Ce système revient à imposer une pression nulle en chaque point de contrôle. Le champ secondaire aura la même amplitude et une phase opposée au champ primaire en $P_{\mathrm{i}} \forall \mathrm{i}$.

Mais ce système n'aura qu'un seul ensemble de $\mathrm{J}$ solutions, correspondant aux $\mathrm{J}$ commandes des sources secondaires, $q u^{\prime}$ à condition que $\mathrm{I}=\mathbf{J}$.

$a$ en résolvant l'équation $B=0$

Ce système permet d'obtenir un ensemble de J solutions de la forme :

$$
[\mathrm{U}]=-\left[\mathrm{H}^{*} \cdot \mathrm{H}\right]^{-1} \cdot\left[\mathrm{H}^{*}\right] \cdot[\mathrm{P}(\mathrm{p})] \quad \text { avec } \mathrm{J} \leq \mathrm{I} \text {. }
$$

* matrice transposée conjuguée

L'application des commandes aux J sources secondaires donnera aux I points de contrôle une pression acoustique résultante non nulle, mais dont l'énergie globale sera minimisée.

\section{Exploitation du code.}

\subsection{Méthodologie.}

\subsubsection{Calcul de rayonnement.}

Cette phase consiste à définir les différentes sources à chaque fréquence, définir le maillage de la géométrie et calculer le champ rayonné par chacune des sources aux points de contrôle et aux points d'observation. A ce stade, les sources secondaires sont caractérisées par une pression de $1 \mathrm{~Pa}(\varphi=0)$. 3.1.2 Minimisation.

Cette étape permet de calculer, pour une configuration donnée, les commandes en amplitude et phase des sources secondaires permettant une minimisation de l'énergie acoustique sur l'ensemble des points de contrôle.

\subsubsection{Recomposition.}

Ces derniers calculs permettent de faire la sommation cohérente, aux points d'observation, des contributions en pression des différentes sources. A ce stade, les sources secondaires sont 
renseignées avec les commandes en pression calculées lors de l'étape de minimisation. Comme seuls les effets linéaires de l'acoustique sont pris en compte, l'évolution en amplitude et en phase d'une contribution $P_{i j}{ }^{n}, \forall i$, est celle imposée par la commande $U_{j}$ associée à la jième source secondaire. Il n'est donc pas nécessaire de recalculer le champ rayonné par chacune des sources avec leur commande respective, mais seulement d'appliquer la correction directement au champ calculé initialement.

\subsection{Objectif.}

L'objectif principal est de pouvoir maîtriser la directivité du rayonnement acoustique d'un moteur d'avion, en vue de réduire, dans des directions privilégiées, les contraintes sonores. Une des grandes inconnues reste le transfert d'un CAA en champ proche, au sens géométrique du terme, vers un champ lointain, sachant que le champ acoustique à traiter est d'une grande richesse modale.

\subsection{Application.}

L'observation des résultats se fait en scindant le domaine d'observation en quatre quadrants. Il est donc recherché une réduction de la puissance acoustique dans un seul des quadrants, choisi à l'avance. La qualité des résultats se juge en terme de GAIN (valeur de la réduction de puissance, en $\mathrm{dBw}$, dans le quadrant voulu) et de COUT (puissance du champ secondaire à fournir par rapport à la puissance du champ primaire). Un fort gain à faible coût est bien sûr ce que l'on peut espérer de mieux.

Les principales études menées jusqu'à présent à SNECMA; tant sur le plan numérique qu'expérimental, ont permis de montrer une réelle capacité du CAA à modifier la directivité d'un champ acoustique. Pour cela, il n'est d'ailleurs pas forçément utile de déployer de gros moyens, en terme de nombre de sources secondaires ou de points de contrôle. Un gain de $8 \mathrm{~dB}$ en puissance dans le bon quadrant pour un coût nul (entendre par là un champ secondaire de même puissance que le champ primaire) est tout à fait réalisable [3]. Par contre, il peut y avoir renforcement de la puissance dans les autres quadrants, ce qui ne constitue pas forcément un problème pour notre application.

\section{Conclusion.}

L'ERASCA permet déjà de fournir de précieux renseignements sur la maîtrise par CAA de la directivité d'un champ acoustique, mais il est nécessaire d'aller plus loin :

on validant le code sur la base des derniers essais réalisés.

a en optimisant I'ERASCA pour en obtenir une version industrielle, capable d'explorer et de mettre au point les solutions les plus efficaces.

5. Bibliographie.

[1] M.A. HAMDI : Formulation variationnelle par équation intégrale pour le calcul de champs acoustiques proches et lointains - Thèse de docteur d'état UTC France 1982.

[2] L. MEBAREK : Méthode numérique par éléments finis de frontière pour le calcul par équations intégrales de champs acoustiques rayonnés par des structures axisymétriques.

Thèse de docteur d'état - UTC France 1986.

[3] D. BERGE - E. BOUTY - J.M. CAILLEAU : Active noise control of a jet engine at low frequency radiation : B.E.M. for predicting far- field noise reduction - AIAA 93-4354.

\section{Notations.}

I nombre de points de contrôle (indice: $\mathrm{i}=1, \mathrm{I}$ )

$\mathrm{J}$ nombre de sources secondaires (indice: $\mathrm{j}=1, \mathrm{~J}$ )

$f_{o} \quad$ fréquence fondamentale.

$\stackrel{\circ}{N}$ nombre d'harmoniques pris en compte (indice: $n=1, N$ )

$\mathbf{P}_{\mathrm{i}}^{\mathrm{n}} \quad$ pression au point de contrôle $\mathrm{i}$ à la fréquence $n . \mathrm{f}_{\mathrm{o}}$ de la source primaire.

$\mathbf{P}_{\mathrm{ij}}{ }^{\mathrm{n}} \quad$ pression au point de contrôle $\mathrm{i}$ à la fréquence $n . \mathrm{f}_{\mathrm{o}}$ de la source secondaire $\mathrm{j}$.

$\mathrm{U}_{j}^{\mathrm{n}} \quad$ pression imposée caractérisant la source secondaire $\mathrm{j}$ à la fréquence $n . f_{o}$.

$H_{i j}{ }^{n} \quad$ fonction de transfert complexe de la source secondaire $j$ vers le point $i$, à la fréquence $n . f_{o}$. 